\title{
The velocity distribution of young radio pulsars: simulating the observations
}

\author{
J.W. Hartman \\ Astronomical Institute, Utrecht, the Netherlands
}

\section{Introduction}

Lyne \& Lorimer (1994) argue that the velocities of young pulsars are much higher than was previously thought. However, recent radio pulsar population synthesis work by Hartman et al. (1996, these proceedings) shows that simulations that use a birth velocity distribution with more pulsars at low velocities, Phinney's modification of the Paczyński (1990) distribution

$$
p(u) d u=\frac{4}{\pi} \frac{d u}{\left(1+u^{2}\right)^{2}}, u=\frac{v_{\mathbf{i}}}{\sigma_{v}}, \sigma_{v} \simeq 600 \mathrm{~km} / \mathrm{s}
$$

describes the observed magnetic field, period and luminosity distribution equally well as simulations that use the Lyne \& Lorimer distribution.

We use this radio pulsar population synthesis model to obtain the proper motion distribution of the simulated sample, and from that we calculate the transverse velocity distribution to compare it with the observed transverse velocity distribution. A full report of this work will be given in Hartman (1996).

\section{Calculations}

To be consistent in our analysis, we apply the same selection effects to the observed sample as we do on our simulated sample. Therefore we have to remove from the observed sample those pulsars that have a distance projected onto the galactic plane larger that $4 \mathrm{kpc}$. This leaves us with 26 pulsars from the 29 pulsars with a characteristic age less than $3 \mathrm{Myr}$ that Lyne \& Lorimer have used to determine the velocity distribution. (Keeping all 29 pulsars does not change our conclusions).

In the left panel of figure 1 we show the resulting cumulative transverse velocity distributions for the Lyne \& Lorimer and Phinney-Paczyński velocity distribution. The Lyne \& Lorimer distribution fits the observations better than the Phinney-Paczyński distribution.

However, we have not taken into account the large errors that affect proper motion measurements. We fit the error distribution of the 94 proper motion measurements that are present in the last published version of the Princeton catalog (Taylor et al. 1993). From this error distribution we randomly draw an error and assign it to the simulated proper motions, and obtain a new transverse velocity from that. The main effect of this procedure is to increase the lowest velocities. The resulting transverse velocity distribution is shown in the right panel of figure 1. Clearly, the Phinney-Paczyński velocity distribution now fits 

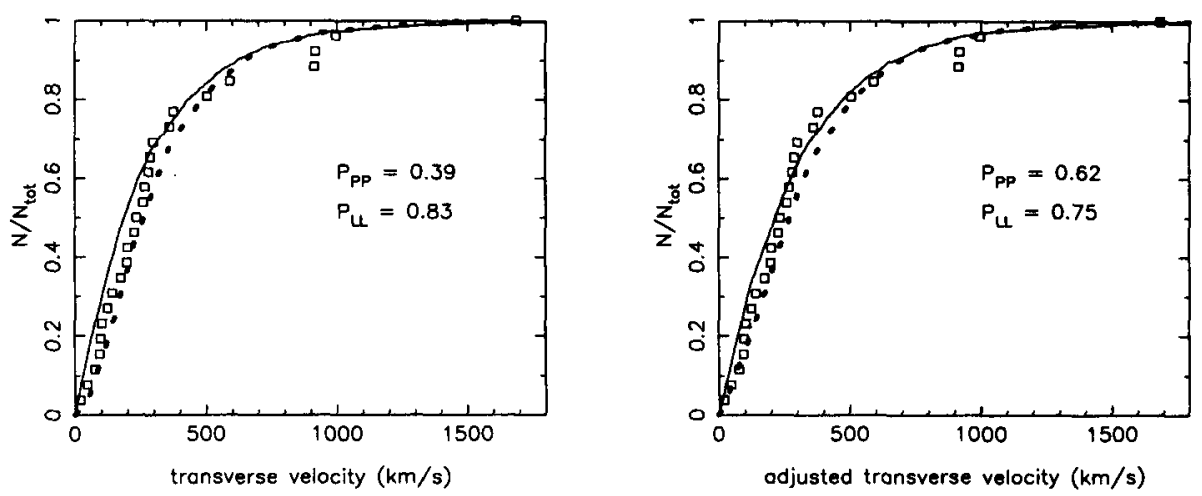

Figure 1. On the left the cumulative transverse velocity distribution of 26 real pulsars from Lyne \& Lorimer (1994) (open squares), and the simulated transverse velocity distributions calculated with the Lyne \& Lorimer (1994) distribution (dotted line) and the Phinney-Paczyński distribution (solid line). The Kolmogorov-Smirnov probability that the simulated distribution and the observed distribution are drawn from the same mother distribution is written in the diagram.

On the right the same distributions, but now after adjusting the simulated transverse velocity distributions for errors in the proper motion measurements.

the observation better than it did previously. We must keep in mind however, that these calculations are based on an observed sample consisting of only 26 pulsars.

We conclude that it is quite possible that the pulsar population contains a large fraction of low velocity pulsars. More proper motion measurements with higher accuracy are needed to provide conclusive data to more precisely determine the shape of the velocity distribution of young pulsars. For instance, one could re-measure with greater accuracy the slowest pulsars in the LyneLorimer data set and check whether the more accurate values are lower on average.

\section{References}

Bhattacharya, D., Wijers, R., Hartman, J., Verbunt, F. 1992, A\&A, 254, 198 Hartman, J., 1996, A\&A in prep.

Lyne, A., Lorimer, D. 1994, Nat, 369, 127

Paczyński, B. 1990, ApJ, 348, 485

Taylor, J., Manchester, R., Lyne, A. 1993, ApJS, 88, 529 